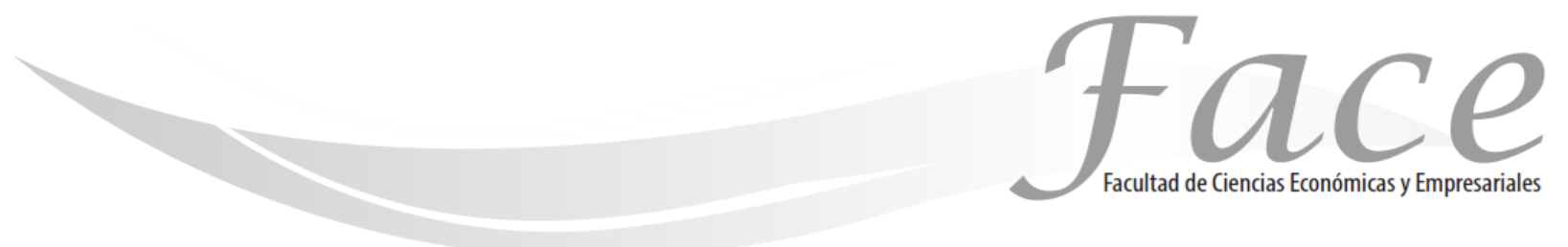

ISSN Impreso: 1794-9920

ISSN Electrónico: 2500-9338

Volumen $19-\mathrm{N}^{\circ} 2$

Año 2019

Págs. $16-23$

(c) (i) $\odot$

\title{
EL DESVANECIMIENTO DEL HUMANISMO EN LAS ORGANIZACIONES
}

\author{
Juan Bernardo Amezcua-Núñez * \\ ORCID: https://orcid.org/0000-0003-1461-4962 \\ Alicia de la Peña-de León** \\ ORCID: https://orcid.org/0000-0001-6338-2067 \\ Juana María Saucedo Soto *** \\ ORCID: https://orcid.org/0000-0002-8075-8764
}

Fecha de Recepción: Julio 5 de 2019

Fecha de Aprobación: Octubre 10 de 2019

\section{Resumen:}

El presente trabajo de investigación busca evaluar la vigencia de los preceptos de la teoría humanista en las empresas o si, por el contrario, esta teoría perdió terreno ante los esfuerzos de las compañías por disminuir sus costos y maximizar las utilidades, siguiendo las enseñanzas que dejó la administración científica en relación con la simplificación de tareas y la automatización de procesos. El artículo es una revisión de literatura de autores recientes y algunos textos originales clásicos manteniendo la siguiente estructura. Primero se realiza una breve descripción de la naturaleza de las organizaciones y del vínculo formado con la administración científica. En una segunda parte se analiza el surgimiento del humanismo y de las teorías de la motivación de los individuos en las organizaciones. En la tercera parte se describen los puntos de coincidencia entre el humanismo y la administración científica que nos permiten considerar que no son modelos tan opuestos al menos en lo que respecto a lo establecido por el humanismo clásico. Posteriormente se hacen algunos cuestionamientos al modelo humanista para terminar con un análisis que a manera de conclusión nos permite asegurar que el humanismo se ha desvanecido dentro de las organizaciones. Desde el punto de vista de teorías sustentables, el humanismo no es recomendable si se parte de la premisa de que las necesidades del ser humano son ilimitadas, pues irían en perjuicio del medio ambiente. Para las nuevas teorías, el ser humano no puede estar por encima de los recursos naturales, pues constituyen una especie de suicidio. Se deben acotar los derechos de los individuos para respetar los derechos del lugar en que vivimos.

Palabras clave: Humanismo, administración científica, responsabilidad social corporativa

* Doctor en Ciencias Administrativas. Profesora Investigadora de la Facultad de Mercadotecnia, Universidad Autónoma de Coahuila, México. Universidad Autónoma de Coahuila Universidad Autónoma de Coahuila Mexico . Contacto: mauricio.ortigosa@anahuac.mx

* * Doctora en Ciencias Administrativas. Profesora Investigadora de la Facultad de Mercadotecnia, Universidad Autónoma de Coahuila, México. Universidad Autónoma de Coahuila Universidad Autónoma de Coahuila Mexico Contacto: mktgheraldo@yahoo.com.mx

** * Doctora de la Universitat Politècnica de Catalunya (UPC), España. Profesora Investigadora de la Facultad de Mercadotecnia, Universidad Autónoma de Coahuila, México. Universidad Autónoma de Coahuila Universidad Autónoma de Coahuila Mexico. Contacto: Jsaucedo62@hotmail.com, 


\title{
THE FADING OF HUMANISM IN ORGANIZATIONS
}

\begin{abstract}
:
The present research seeks to assess the validity of the precepts of humanistic theory in companies or if, on the contrary, this theory lost ground to the efforts of companies to reduce their costs and maximize profits, following the teachings that left the scientific administration in relation to the simplification of tasks and the automation of processes. The article is a literature review of recent authors and some classic original texts maintaining the following structure. First, a brief description of the nature of the organizations and the link formed with the scientific administration is made. In a second part the emergence of humanism and the theories of the motivation of individuals in organizations is analyzed. The third part describes the points of agreement between humanism and scientific administration that allow us to consider that they are not so opposed models, at least as regards what is established by classical humanism. Subsequently some questions are made to the humanist model to end with an analysis that in a way allows us to ensure that humanism has vanished within organizations. From the point of view of sustainable theories, humanism is not advisable if one starts from the premise that the needs of the human being are unlimited, because they would harm the environment. For new theories, the human being can not be above natural resources, because they constitute a kind of suicide. The rights of individuals must be limited to respect the rights of the place where we live
\end{abstract}

Keywords: Humanism, scientific administration, corporate social responsibility.

\section{O DESVANECIMENTO DO HUMANISMO NAS ORGANIZAÇÕES}

\section{Resumo:}

A presente pesquisa busca avaliar a validade dos preceitos da teoria humanística nas empresas ou se, pelo contrário, essa teoria perdeu espaço para os esforços das empresas em reduzir seus custos e maximizar lucros, seguindo os ensinamentos que deixaram a administração científica em relação às empresas. à simplificação de tarefas e à automação de processos. 0 artigo é uma revisão de literatura de autores recentes e alguns textos originais clássicos, mantendo a seguinte estrutura. Primeiro, é feita uma breve descrição da natureza das organizações e o vínculo formado com a administração cientíica. Numa segunda parte, analisa-se o surgimento do humanismo e as teorias da motivação dos indivíduos nas organizações. A terceira parte descreve os pontos de concordância entre humanismo e administração científica que nos permitem considerar que eles não são modelos tão opostos, pelo menos no que diz respeito ao que é estabelecido pelo humanismo clássico. Posteriormente, algumas perguntas são feitas ao modelo humanista para terminar com uma análise que de certa forma nos permite garantir que o humanismo desapareça dentro das organizações. Do ponto de vista das teorias sustentáveis, o humanismo não é aconselhável se partirmos da premissa de que as necessidades do ser humano são ilimitadas, porque prejudicariam o meio ambiente. Para novas teorias, o ser humano não pode estar acima dos recursos naturais, porque constitui uma espécie de suicídio. Os direitos dos indivíduos devem ser limitados para respeitar os direitos do local em que vivemos.

Palavras-chave: Humanismo, administração científica, responsabilidade social corporative. 


\section{INTRODUCCIÓN:}

El presente trabajo de investigación busca evaluar la vigencia de los preceptos de la teoría humanista en las empresas o si, por el contrario, esta teoría perdió terreno ante los esfuerzos de las compañías por disminuir sus costos y maximizar las utilidades, siguiendo las enseñanzas que dejó la administración científica en relación con la simplificación de tareas y la automatización de procesos.

El artículo es una revisión de literatura de autores recientes y algunos textos originales clásicos manteniendo la siguiente estructura. Primero se realiza una breve descripción de la naturaleza de las organizaciones y del vínculo formado con la administración científica. En una segunda parte se analiza el surgimiento del humanismo y de las teorías de la motivación de los individuos en las organizaciones. En la tercera parte se describen los puntos de coincidencia entre el humanismo y la administración científica que nos permiten considerar que no son modelos tan opuestos al menos en lo que respecto a lo establecido por el humanismo clásico. Posteriormente se hacen algunos cuestionamientos al modelo humanista para terminar con un análisis que a manera de conclusión nos permite asegurar que el humanismo se ha desvanecido dentro de las organizaciones.

\section{MARCO TEORICO}

\section{Las Organizaciones y las Teorías Clásicas de la Administración}

Las organizaciones y empresas constituyeron un campo fértil para el estudio científico de la administración durante los últimos 100 años. La "nueva" disciplina administrativa impulsada principalmente por la actual Universidad CarnegieMellon y la Universidad de Columbia, nació como un proyecto multidisciplinario donde trabajaban científicos, economistas y psicólogos con el fin de analizar lo que llevaba a los empleados a ser más productivos (Scott, 2004).

De acuerdo con Barba (2007), desde un inicio, en esta nueva disciplina se identificaron tres corrientes dentro del pensamiento organizacional: la mecánica, la orgánica y la cultural. La corriente mecánica corresponde a la época de los adelantos en la ciencia física y dio una visión del hombre como una máquina que debía ser analizada bajo los principios de la mecánica. El hombre, como la máquina, es considerado capaz de realizar tareas rutinarias con eficiencia y la labor de los administradores consiste en identificar formas de incrementar la productividad mediante el ajuste físico de las labores realizadas por el hombre-máquina. El pensamiento orgánico en contraparte se inspiró en los procesos de la biología para hacer analogías entre las organizaciones (cuerpo social) y el cuerpo humano, definiendo la función que debiera desempeñar el hombre-célula dentro del "organismo". Y, por último, el análisis de la cultura revisó el significado simbólico de las acciones del hombre, de la organización y su interacción con el medio ambiente como el medio más efectivo para aumentar la productividad de la empresa.

En este contexto y sin importar la perspectiva elegida, la administración científica encontró un campo idóneo donde continuar los estudios relacionados con la productividad y la eficiencia. Surgieron así, los clásicos de la administración como Taylor, Gantt, Fayol y Weber con interés en la división de las tareas, análisis del área de trabajo, mejoramiento de las herramientas y la motivación de los trabajadores para mejorar el desempeño económico en las organizaciones (Pindur, Rogers y Kim, 1995).

Analizar y mejorar las tareas del trabajador se convirtió en un punto central. A la "máquina humana" como la consideraba Taylor, había que comunicarle 
cómo hacer su trabajo de la mejor manera física y psicológica. A un aumento en su productividad el empleado obtenía un incentivo económico (Fleischman, 2000). Así la motivación extrínseca, es decir el conjunto de recompensas monetarias directas (e.g., salario, bonos, complementos por méritos) e indirectas (e.g., tiempo no trabajado, programas de protección y pagos en especie) que recibía un individuo por su trabajo se consideró necesaria para que el trabajador llevara a cabo tareas valiosas para la organización (Osterloh y Frey, 2000).

El punto de discusión surgió al considerar que la mano de obra era simultáneamente un recurso económico y un ser humano social (Shields y Grant, 2010). Esta doble acepción permitió el surgimiento de la teoría de las relaciones humanas dentro de las organizaciones.

\section{El Humanismo en las Organizaciones}

Pindur et al. (1995) señalan a Mary Parker Follet como una de las primeras aportadoras en el estudio del comportamiento humano dentro de las organizaciones al identificar el conflicto entre la administración y el empleado como una base para el desarrollo de las mismas. De igual forma los autores destacan las aportaciones de Mayo, Maslow, Herzberg, Simon, McGregor y Argyris en la consolidación del modelo humanista que debían seguir las organizaciones.

Esta primera ola del humanismo, ahora reconocidos como clásicos, elaboró propuestas para identificar aquellas necesidades de los trabajadores que les proporcionaran satisfacción en el trabajo y por ende aumentaran su productividad. Identificaron una serie de necesidades básicas que eran cubiertas por los motivadores extrínsecos y otro grupo de necesidades de mayor rango entre las que se encontraban la aceptación social y la auto-realización, catalogadas como motivadores intrínsecos (Pindur et al., 1995)
Investigadores académicos y hombres de negocios como Chester Barnard analizaron la problemática interna de las organizaciones con un enfoque más amplio que el puramente económico, que buscaba entender las relaciones de los empleados con las figuras de autoridad y con sus compañeros de trabajo (Williamson, 1981). El mayor aporte del estudio del comportamiento humano dentro de las organizaciones fue su contribución para entender las motivaciones, las dinámicas de grupos y el liderazgo, logrando atraer la atención de la administración hacia estos procesos y reforzar la idea de que los empleados son un recurso valioso (Pindur et al., 1995).

\section{Coincidencias entre el Humanismo y las Teorías Económicas}

Aunque la consideración de las relaciones humanas surgió como una reacción contra el razonamiento frío y mecánico de la administración científica, ambos modelos comparten dos situaciones básicas. Por un lado, ambas perspectivas buscan incrementar el esfuerzo de los trabajadores en su trabajo y por el otro asumen que los intereses de los trabajadores están alineados con los de la organización (Kaplan y otros, 1972). Incluso Taylor sentía que la administración científica reducía el conflicto entre patrón y empleados, pues si el administrador sabía cómo hacer un buen trabajo le podía hacer una demostración al trabajador (Fleischman, 2000).

En la actualidad, aún las empresas autoproclamadas como humanistas siguen empleando los principios de la administración científica. Vallas (1999) encontró que los métodos de estandarización y control tiene un gran auge, especialmente si analizamos los sistemas de franquicias y las industrias de la transformación. El taylorismo sigue vigente en un gran número de empresas del estilo de McDonalds, donde los 
métodos de trabajo son predecibles y controlables (Ritzer, 1993). Así mismo, las empresas productoras altamente automatizadas aplican el principio de mantener las tareas tan simples que no requieran habilidades especiales de los trabajadores (Prujit, 2000). Las tareas simples y rutinarias, sin duda incrementan la productividad, pero a costa de los motivadores intrínsecos como la satisfacción por el trabajo realizado y la autorealización.

\section{Críticas al humanismo}

Existen opiniones que consideran que el humanismo y el utilitarismo de la administración científica no deben mezclarse, pues al tratar de incorporarlos en una organización, sólo se corrompen ambos modelos (Morrow y Thayer, 1978). El humanismo ha permanecido unido a la idea de la organización por más de 100 años. Sin embargo, existen autores que lo consideran como un concepto generado por la clase dominante para su provecho personal (Barba, 2007). La generación de un sistema productivo basado en el reconocimiento social para lograr la satisfacción del empleado, es una estrategia de lograr altos niveles de productividad sin aumentarle el sueldo ni proporcionarle otros beneficios económicos (Allyn y Thayer, 1978).

El humanismo no glorifica al ser humano. Al contrario, autores como March y Simon (1987) reconocen que los empleados son instrumentos pasivos que aceptan órdenes para realizar un trabajo, requieren de un líder autocrático que los dirija -debido a su incapacidad para tomar decisiones en la organización- (Bruce y Nyland, 2011) y consideran no sólo al individuo como incapaz de razonar, sino al grupo entero de trabajadores como una masa irracional que igualmente necesita dirección (Barba, 2007). Esta dirección, alcanza la vida privada de los trabajadores al tratar de imponerles ideologías que defiende la organización o las instituciones con las que ésta se relaciona, lo que supone una intromisión a la vida personal (Coriat, 2001).
Las teorías que tratan de explicar las motivaciones del trabajador no han sido del todo exitosas, en parte por la complejidad que representa estudiar una conducta que refiere a un constructo global cuyo alcance es mayor que la de los elementos que la integran (Schneider y Alderfer, 1973). Schneider aclara que teorías como la de Maslow, que fueron desarrolladas para definir estados y conductas individuales fueron adoptadas para describir las conductas organizacionales lo que ha complicado su implementación. La postura de Maslow que defiende que el individuo está en la permanente búsqueda de la autorrealización, no basta para explicar cómo se comportan los grupos de trabajadores en una organización

Aún más, Scheneider y Alderfer (1973) concluyen que no es posible motivar a los empleados adecuadamente utilizando solamente la jerarquía de las necesidades de Maslow o la teoría de los dos factores de Herzberg (motivadores intrínsecos e extrínsecos) porque dichas teorías asumen que las necesidades humanas son insaciables y por lo tanto nunca se satisfacen (Ver figura 1). Asimismo, en ese sentido, la teoría de la motivación de Elton Mayo, trata de explicar que tanto los factores económicos, salariales y las recompensas, sociales y simbólicas, motivan a los empleados influyendo en su comportamiento (García y Larios-Gómez, 2017). Los seres humanos como centro de las teorías humanistas son vistos como organismos superiores, que están por encima del resto de las especies que pueblan al planeta. Por tal motivo se priman sus derechos sobre las necesidades de la empresa e incluso del planeta. Para los humanistas recalcitrantes, las organizaciones existen por y para los individuos. 
Figura 1.

Orientación científica Vs Humanista según los motivadores individuales según Maslow y Herzberg

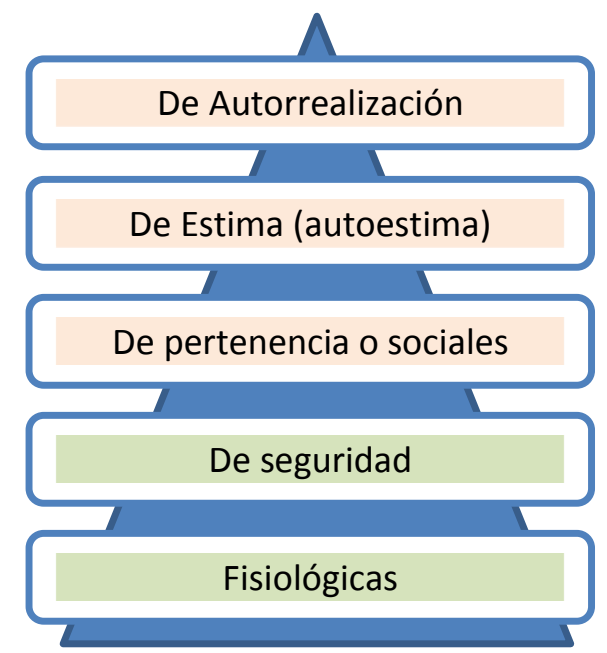

\begin{tabular}{|l|l|l|}
\hline $\begin{array}{l}\text { Factores } \\
\text { Motivacionales (De } \\
\text { satisfacción con el } \\
\text { contenido del trabajo) }\end{array}$ & $\begin{array}{l}\text { Factores de Higiene (De } \\
\text { insatisfacción con el } \\
\text { contexto del trabajo) }\end{array}$ \\
\hline \begin{tabular}{llll|} 
1. El trabajo en sí & 1. Condiciones del trabajo \\
\hline 2. Realización & $\begin{array}{l}\text { 2.Administración de la } \\
\text { organización }\end{array}$ \\
\hline 3. Reconocimiento & $\begin{array}{l}\text { 3. Salario } \\
\text { 4. Progreso } \\
\text { profesional }\end{array}$ & $\begin{array}{l}\text { 4. Relaciones con el } \\
\text { supervisor }\end{array}$ \\
\hline 5. Responsabilidad & 5. Beneficios sociales \\
\hline Orientación humanista & Orientación científica \\
\hline
\end{tabular} \\
\hline
\end{tabular}

Fuente: elaboración propia.

Sin embargo, para otras corrientes de pensamiento filosófico, los individuos son sólo un componente más de este mundo, y por tal razón sus derechos deben ser resguardados en igual proporción al de otros seres vivos. En apoyo a esta idea, el pensamiento ecológico contemporáneo de la administración sostiene que la devastación de nuestro planeta se deriva de la sobrevaloración de lo humano en perjuicio de la naturaleza (Barba, 2007).

Para Barba (2007) el bienestar humano no debe estar por encima del bienestar de nuestro planeta. Las acciones de las empresas, y por lo tanto la de los individuos que la componen, deben estar limitadas por el bienestar del mundo en su totalidad. La productividad de las empresas por lo tanto no debe ser prioridad, si esto implica exprimir a los individuos que la conforman o el contexto (naturaleza) en que opera.
Sin embargo, el análisis de este trabajo se ha enfocado en dirimir si el humanismo es central para un buen desempeño de las organizaciones en nuestros tiempos. La pregunta es si ¿es necesario siquiera motivar a los empleados? En estudios realizados por Kaplan y otros (1972) a obreros y gerentes de nivel medio en varias empresas de Estados Unidos se encontró que existe un apego natural del individuo hacia su trabajo. Es parte de su esencia como ser humano. Al preguntarles si trabajarían aún si tuvieran el dinero para vivir cómodamente el resto de su vida, el $80 \%$ contestó que sí. Estar en casa sin hacer nada no parece una buena idea para ellos. Este apego según explica Kaplan puede deberse a motivos sociales más que por los económicos. Los individuos crean comunidades con sus compañeros de trabajo. 


\section{CONCLUSIONES:}

El humanismo nació como una reacción al trato utilitarista que la administración científica les concedió a los empleados en la búsqueda de maximizar la productividad en las organizaciones. A pesar de que la administración científica cayó en desuso, actualmente muchas empresas siguen empleando técnicas que les permiten disminuir sus costos y aumentar sus utilidades sin considerar el efecto en la vida de sus trabajadores.

La automatización de los procesos ha permitido a las organizaciones depender menos de la mano de obra, lo que lleva a prácticas que poco tienen de humanistas como son los recortes de trabajadores, baja de sueldos en lugar de despido o relocalización de plantas. Los países que cuentan con leyes más laxas para la protección de los trabajadores son los que captan la mayor inversión de las grandes fábricas. Sin mencionar, algunas plantas donde las líneas de producción son operadas al 100\% por robots.

Aún desde el punto de vista de teorías sustentables, el humanismo no es recomendable si se parte de la premisa de que las necesidades del ser humano son ilimitadas, pues irían en perjuicio del medio ambiente. Para las nuevas teorías, el ser humano no puede estar por encima de los recursos naturales, pues constituyen una especie de suicidio. Se deben acotar los derechos de los individuos para respetar los derechos del lugar en que vivimos.
Sin embargo, no podemos negar la prevalencia de una conciencia comunitaria que busca humanizar el mundo empresarial. Donde se considera a la empresa como un punto de partida para logar una sociedad más humana. Entendiendo por "más humana" como una sociedad que prioriza y protege a los individuos. Pero la vigencia del tema, no necesariamente refleja la realidad al interior de las organizaciones. Es políticamente correcto proyectar una imagen humanista, donde el empleado es el centro mismo de la organización a la vez que se respeta el medio ambiente. Pero las políticas de crecimiento de las organizaciones globales, no necesariamente van en la misma dirección. Parece que existe un mensaje contradictorio de las organizaciones; pues hacia el exterior se habla del valor del humanismo, pero al interior de sus puertas este concepto parece que se ha desvanecido.

En futuras investigaciones, será importante abordar el cambio que ha tenido el humanismo en organizaciones que ahora funcionan como sistemas abiertos, con contextos de trabajo tan diferentes como el home office y la economía colaborativa.

\section{REFERENCIAS:}

Barba, A. (2007). Humanismo y organización: los dilemas de una reflexión. Administración y Organizaciones, 9(18), 31-47.

Bruce, K., Nyland, C. (2011). Elton Mayo and the deification of human relations. Organization Studies, 32(3), 383-405.

Coriat, B. (2001). El Taller y el cronómetro: ensayo sobre el taylorismo, el fordismo y la producción en masa. Madrid: Siglo XXI

Fleischman, R. K. (2000). Completing the triangle: taylorism and the paradigms. Accounting, Auditing \& Accountability Journal, 13(5), 597-623. 
Garcia, G., \& Larios-Gómez, E. (2017). Capítulo 4 Análisis del clima laboral de la Ibero-Puebla. En Administración. Un enfoque latinoamericano México-Brasil Coord. Larios-Gómez, E., \& Giuliani, A. C., 2017. ISBN LIBRO IMPRESO: 978-607-32-4000-0. ISBN E-BOOK: 978-607-324005-5 Editorial Pearson : México.

Kaplan, H. R. y O. (1973, Agosto). Human relations perspectives on motivation: a critical appraisal. Presentado en la reunión de la asociación americana de sociología, Nueva Orleans, Louisiana.

March, J. G., Simon, H. A. (1987). Teoría de la organización. Recuperado de Bibliotecas del Tecnológico de Monterrey.

Morrow, A. A., Thayer, F. (1978) Materialism and humanism: organization theory's odd couple. Administration and Society, 10, 86-106. doi: 10.1177/009539977801000104

Osterloh , M. y Frey, B. (2000). Motivation, knowledge transfer and organizational forms. Organization Science, 11(5), 538-550.

Pindur, W., Rogers, S. E., y Kim, P. S. (1995). The history of management: a global perspective. Journal of Management History. 1(1), 59-77.

Prujit, H. (2000). Repainting, modifying, smashing taylorism. Journal of Organizational Change Management, 13(5), 439-451.

Ritzer, G. (1993), The mcdonaldization of society: an investigation into the changing character of contemporary social life, Thousand Oaks, CA Pine Forge Press.

Scott, W. R. (2004). Reflections on a half-century of organizational sociology. Annual Review of Sociology, $\quad 30, \quad 1-21$. doi:10.1146/annurev.soc.30.012703.110644

Shields, J., Grant, D. (2010). Psychologising the subject: hrm, commodification and the objectification of labour. Economic and Labour Relations Review, 20(2), 61-76.

Schneider, B., Alderfer, C. P. (1973) Three studies of measures of need satisfaction in organizations. Administrative Science Quaterly, 18(4), 489-505.

Vallas, S. P. (1999). Rethinking post-fordism: the meaning of workplace flexibility, Sociological Theory, 17, 78-101.

Williamson, O. E. (1981). The modern corporation: origins, evolution, attributes. Journal of Economic Literature, 19, 1537-1568 